\title{
On the basis property of the root functions of some class of non-self-adjoint Sturm-Liouville operators
}

Cemile Nur ${ }^{*}$ and Oktay A Veliev

\section{"Correspondence:}

cnur@dogus.edu.tr

Department of Mathematics, Dogus

University, Acıbadem, Kadiköy,

Istanbul, Turkey

\begin{abstract}
We obtain the asymptotic formulas for the eigenvalues and eigenfunctions of the Sturm-Liouville operators with some regular boundary conditions. Using these formulas, we find sufficient conditions on the potential $q$ such that the root functions of these operators do not form a Riesz basis.
\end{abstract}

MSC: 34L05; 34L20

Keywords: asymptotic formulas; regular boundary conditions; Riesz basis

\section{Introduction and preliminary facts}

Let $T_{1}, T_{2}, T_{3}$, and $T_{4}$ be the operators generated in $L_{2}[0,1]$ by the differential expression

$$
l(y)=-y^{\prime \prime}+q(x) y
$$

and the following boundary conditions:

$$
\begin{aligned}
& y_{0}^{\prime}+\beta y_{1}^{\prime}=0, \quad y_{0}-y_{1}=0 \\
& y_{0}^{\prime}+\beta y_{1}^{\prime}=0, \quad y_{0}+y_{1}=0 \\
& y_{0}^{\prime}-y_{1}^{\prime}=0, \quad y_{0}+\alpha y_{1}=0
\end{aligned}
$$

and

$$
y_{0}^{\prime}+y_{1}^{\prime}=0, \quad y_{0}+\alpha y_{1}=0
$$

respectively, where $q(x)$ is a complex-valued summable function on $[0,1], \beta \neq \pm 1$ and $\alpha \neq$ \pm 1 .

In conditions (2), (3), (4), and (5) if $\beta=1, \beta=-1, \alpha=1$, and $\alpha=-1$, respectively, then any $\lambda \in \mathbb{C}$ is an eigenvalue of infinite multiplicity. In (2) and (4) if $\beta=-1$ and $\alpha=-1$ then they are periodic boundary conditions; in (3) and (5) if $\beta=1$ and $\alpha=1$ then they are antiperiodic boundary conditions.

These boundary conditions are regular but not strongly regular. Note that, if the boundary conditions are strongly regular, then the root functions form a Riesz basis (this result was proved independently in $[1,2]$ and [3]). In the case when an operator is associated

(02014 Nur and Veliev; licensee Springer. This is an Open Access article distributed under the terms of the Creative Commons Attribution License (http://creativecommons.org/licenses/by/2.0), which permits unrestricted use, distribution, and reproduction in any medium, provided the original work is properly cited. 
with the regular but not strongly regular boundary conditions, the root functions generally do not form even a usual basis. However, Shkalikov $[4,5]$ proved that they can be combined in pairs, so that the corresponding 2-dimensional subspaces form a Riesz basis of subspaces.

In the regular but not strongly regular boundary conditions, periodic and antiperiodic boundary conditions are the ones more commonly studied. Therefore, let us briefly describe some historical developments related to the Riesz basis property of the root functions of the periodic and antiperiodic boundary value problems. First results were obtained by Kerimov and Mamedov [6]. They established that, if

$$
q \in C^{4}[0,1], \quad q(1) \neq q(0),
$$

then the root functions of the operator $L(q)$ form a Riesz basis in $L_{2}[0,1]$, where $L(q)$ denotes the operator generated by (1) and the periodic boundary conditions.

The first result in terms of the Fourier coefficients of the potential $q$ was obtained by Dernek and Veliev [7]. They proved that if the conditions

$$
\begin{aligned}
& \lim _{n \rightarrow \infty} \frac{\ln |n|}{n q_{2 n}}=0, \\
& q_{2 n} \sim q_{-2 n}
\end{aligned}
$$

hold, then the root functions of $L(q)$ form a Riesz basis in $L_{2}[0,1]$, where $q_{n}=:\left(q, e^{i 2 \pi n x}\right)$ is the Fourier coefficient of $q$ and everywhere, without loss of generality, it is assumed that $q_{0}=0$. Here $(\cdot, \cdot)$ denotes the inner product in $L_{2}[0,1]$ and $a_{n} \sim b_{n}$ means that $a_{n}=$ $O\left(b_{n}\right)$ and $b_{n}=O\left(a_{n}\right)$ as $n \rightarrow \infty$. Makin [8] improved this result. Using another method he proved that the assertion on the Riesz basis property remains valid if condition (7) holds, but condition (6) is replaced by a less restrictive one: $q \in W_{1}^{s}[0,1]$,

$$
q^{(k)}(0)=q^{(k)}(1), \quad \forall k=0,1, \ldots, s-1
$$

holds and $\left|q_{2 n}\right|>c n^{-s-1}$ with some $c>0$ for sufficiently large $n$, where $s$ is a nonnegative integer. Besides, some conditions which imply the absence of the Riesz basis property were presented in [8]. Shkalilov and Veliev obtained in [9] more general results, which cover all results discussed above.

The other interesting results as regards periodic and antiperiodic boundary conditions were obtained in [10-19].

The basis properties of other some operators with regular but not strongly regular boundary conditions are studied in [20-23]. It was proved in [22] that the system of the root functions of the operator generated by (1) and the boundary conditions

$$
\begin{aligned}
& y^{\prime}(1)-(-1)^{\sigma} y^{\prime}(0)+\gamma y(0)=0, \\
& y(1)-(-1)^{\sigma} y(0)=0
\end{aligned}
$$

forms an unconditional basis of the space $L_{2}[0,1]$, where $q(x)$ is an arbitrary complexvalued function from the class $L_{1}[0,1], \gamma$ is an arbitrary nonzero complex number and $\sigma=$ 
0,1 . Kerimov and Kaya $[20,21]$ investigated the basis properties of fourth order differential operators with some regular boundary conditions.

In this paper we prove that if

$$
\lim _{n \rightarrow \infty} \frac{\ln |n|}{n s_{2 n}}=0
$$

where $s_{k}=(q, \sin 2 \pi k x)$, then the large eigenvalues of the operators $T_{1}$ and $T_{3}$ are simple. Moreover, if there exists a sequence $\left\{n_{k}\right\}$ such that (8) holds when $n$ is replaced by $n_{k}$, then the root functions of these operators do not form a Riesz basis.

Similarly, if

$$
\lim _{n \rightarrow \infty} \frac{\ln |n|}{n s_{2 n+1}}=0,
$$

then the large eigenvalues of the operators $T_{2}$ and $T_{4}$ are simple and if there exists a sequence $\left\{n_{k}\right\}$ such that (9) holds when $n$ is replaced by $n_{k}$, then the root functions of these operators do not form a Riesz basis.

Moreover, we obtain asymptotic formulas of arbitrary precision for the eigenvalues and eigenfunctions of the operators $T_{1}, T_{2}, T_{3}$, and $T_{4}$.

\section{Main results}

We will focus only on the operator $T_{1}$. The investigations of the operators $T_{2}, T_{3}$, and $T_{4}$ are similar. It is well known that (see (47a) and (47b) on page 65 of [24]) the eigenvalues of the operators $T_{1}(q)$ consist of the sequences $\left\{\lambda_{n, 1}\right\},\left\{\lambda_{n, 2}\right\}$ satisfying

$$
\lambda_{n, j}=(2 n \pi)^{2}+O\left(n^{1 / 2}\right)
$$

for $j=1,2$. From this formula one can easily obtain the following inequality:

$$
\left|\lambda_{n, j}-(2 \pi k)^{2}\right|=|2(n-k) \pi||2(n+k) \pi|+O\left(n^{\frac{1}{2}}\right)>2 n
$$

for $j=1,2 ; k \neq n ; k=0,1, \ldots$, and $n \geq N$, where $N$ denotes a sufficiently large positive integer, that is, $N \gg 1$.

Let us denote by $T_{j}(0)$ the operator $T_{j}$ when $q(x)=0$. The eigenvalues of the operator $T_{1}(0)$ are $\lambda_{n}=(2 \pi n)^{2}$ for $n=0,1, \ldots$. The eigenvalue 0 is simple and the corresponding eigenfunction is 1 . The eigenvalues $\lambda_{n}=(2 \pi n)^{2}$ for $n=1,2, \ldots$ are double and the corresponding eigenfunctions and associated functions are

$$
y_{n}(x)=\cos 2 \pi n x \quad \text { and } \quad \phi_{n}(x)=\left(\frac{\beta}{1+\beta}-x\right) \frac{\sin 2 \pi n x}{4 \pi n},
$$

respectively. Note that for any constant $c, \phi_{n}(x)+c y_{n}(x)$ is also an associated function corresponding to $\lambda_{n}$, since one can easily verify that it satisfies the equation and boundary conditions for the associated functions. It can be shown that the adjoint operator $T_{1}^{*}(0)$ is associated with the boundary conditions

$$
y_{1}+\bar{\beta} y_{0}=0, \quad y_{1}^{\prime}-y_{0}^{\prime}=0
$$

It is easy to see that 0 is a simple eigenvalue of $T_{1}^{*}(0)$ and the corresponding eigenfunction is $y_{0}^{*}(x)=x-\frac{1}{1+\bar{\beta}}$. The other eigenvalues $\lambda_{n}^{*}=(2 \pi n)^{2}$ for $n=1,2, \ldots$, are double and the 
corresponding eigenfunctions and associated functions are

$$
y_{n}^{*}(x)=\sin 2 \pi n x \text { and } \phi_{n}^{*}(x)=\left(x-\frac{1}{1+\bar{\beta}}\right) \frac{\cos 2 \pi n x}{4 \pi n}
$$

respectively.

Let

$$
\varphi_{n}(x):=\frac{16 \pi n(\beta+1)}{\beta-1} \phi_{n}(x)=\frac{4(\beta+1)}{\beta-1}\left(\frac{\beta}{1+\beta}-x\right) \sin 2 \pi n x
$$

and

$$
\varphi_{n}^{*}(x):=\frac{16 \pi n(\bar{\beta}+1)}{\bar{\beta}-1} \phi_{n}^{*}(x)=\frac{4(\bar{\beta}+1)}{\bar{\beta}-1}\left(x-\frac{1}{1+\bar{\beta}}\right) \cos 2 \pi n x
$$

(see (12) and (13)). The system of the root functions of $T_{1}^{*}(0)$ can be written as $\left\{f_{n}: n \in \mathbb{Z}\right\}$, where

$$
f_{-n}=\sin 2 \pi n x, \quad \forall n>0 \quad \text { and } \quad f_{n}=\varphi_{n}^{*}(x), \quad \forall n \geq 0 .
$$

One can easily verify that it forms a basis in $L_{2}[0,1]$ and the biorthogonal system $\left\{g_{n}: n \in\right.$ $\mathbb{Z}$ \} is the system of the root functions of $T_{1}(0)$, where

$$
g_{-n}=\varphi_{n}, \quad \forall n>0 \quad \text { and } \quad g_{n}=\cos 2 \pi n x, \quad \forall n \geq 0,
$$

since $\left(f_{n}, g_{m}\right)=\delta_{n, m}$.

To obtain the asymptotic formulas for the eigenvalues $\lambda_{n, j}$ and the corresponding normalized eigenfunctions $\Psi_{n, j}(x)$ of $T_{1}(q)$ we use (11) and the well-known relations

$$
\left(\lambda_{N, j}-(2 \pi n)^{2}\right)\left(\Psi_{N, j}, \sin 2 \pi n x\right)=\left(q \Psi_{N, j}, \sin 2 \pi n x\right)
$$

and

$$
\left(\lambda_{N, j}-(2 \pi n)^{2}\right)\left(\Psi_{N, j}, \varphi_{n}^{*}\right)-\gamma_{1} n\left(\Psi_{N, j}, \sin 2 \pi n x\right)=\left(q \Psi_{N, j}, \varphi_{n}^{*}\right),
$$

where

$$
\gamma_{1}=\frac{16 \pi(\beta+1)}{\beta-1},
$$

which can be obtained by multiplying both sides of the equality

$$
-\left(\Psi_{N, j}\right)^{\prime \prime}+q(x) \Psi_{N, j}=\lambda_{N, j} \Psi_{N, j}
$$

by $\sin 2 \pi n x$ and $\varphi_{n}^{*}$, respectively. It follows from (18) and (19) that

$$
\begin{aligned}
& \left(\Psi_{N, j}, \sin 2 \pi n x\right)=\frac{\left(q \Psi_{N, j}, \sin 2 \pi n x\right)}{\lambda_{N, j}-(2 \pi n)^{2}} ; \quad N \neq n, \\
& \left(\Psi_{N, j}, \varphi_{n}^{*}\right)=\frac{\gamma_{1} n\left(q \Psi_{N, j}, \sin 2 \pi n x\right)}{\left(\lambda_{N, j}-(2 \pi n)^{2}\right)^{2}}+\frac{\left(q \Psi_{N, j}, \varphi_{n}^{*}\right)}{\lambda_{N, j}-(2 \pi n)^{2}} ; \quad N \neq n .
\end{aligned}
$$


Moreover, we use the following relations:

$$
\begin{aligned}
& \left(\Psi_{N, j}, \bar{q} \sin 2 \pi n x\right)=\sum_{n_{1}=0}^{\infty}\left[\left(q \varphi_{n_{1}}, \sin 2 \pi n x\right)\left(\Psi_{N, j}, \sin 2 \pi n_{1} x\right)\right. \\
& \left.+\left(q \cos 2 \pi n_{1} x, \sin 2 \pi n x\right)\left(\Psi_{N, j}, \varphi_{n_{1}}^{*}\right)\right], \\
& \left(\Psi_{N, j}, \bar{q} \varphi_{n}^{*}\right)=\sum_{n_{1}=0}^{\infty}\left[\left(q \varphi_{n_{1}}, \varphi_{n}^{*}\right)\left(\Psi_{N, j}, \sin 2 \pi n_{1} x\right)+\left(q \cos 2 \pi n_{1} x, \varphi_{n}^{*}\right)\left(\Psi_{N, j}, \varphi_{n_{1}}^{*}\right)\right], \\
& \left|\left(q \Psi_{N, j}, \sin 2 \pi n x\right)\right|<4 M, \\
& \left|\left(q \Psi_{N, j}, \varphi_{n}^{*}\right)\right|<4 M
\end{aligned}
$$

for $N \gg 1$, where $M=\sup \left|q_{n}\right|$. These relations are obvious for $q \in L_{2}(0,1)$, since to obtain (22) and (23) we can use the decomposition of $\bar{q} \sin 2 \pi n x$ and $\bar{q} \varphi_{n}^{*}$ by the basis (16). For $q \in L_{1}(0,1)$ see Lemma 1 of [25].

To obtain the asymptotic formulas for the eigenvalues and eigenfunctions we iterate (18) and (19) by using (22) and (23). First let us prove the following obvious asymptotic formulas, namely (29), for the eigenfunctions $\Psi_{n, j}$. The expansion of $\Psi_{n, j}$ by the basis (17) can be written in the form

$$
\Psi_{n, j}=u_{n, j} \varphi_{n}(x)+v_{n, j} \cos 2 \pi n x+h_{n, j}(x),
$$

where

$$
\begin{aligned}
& u_{n, j}=\left(\Psi_{n, j}, \sin 2 \pi n x\right), \quad v_{n, j}=\left(\Psi_{n, j}, \varphi_{n}^{*}\right), \\
& h_{n, j}(x)=\sum_{\substack{k=0 \\
k \neq n}}^{\infty}\left[\left(\Psi_{n, j}, \sin 2 \pi k x\right) \varphi_{k}(x)+\left(\Psi_{n, j}, \varphi_{k}^{*}\right) \cos 2 \pi k x\right],
\end{aligned}
$$

and $\varphi_{n}(x), \varphi_{n}^{*}(x)$ are defined in (14) and (15), respectively. Using (20), (21), (24), and (25) one can readily see that there exists a constant $C$ such that

$$
\sup \left|h_{n, j}(x)\right| \leq C\left(\sum_{k \neq n}\left(\frac{1}{\left|\lambda_{n, j}-(2 \pi k)^{2}\right|}+\frac{n}{\left|\left(\lambda_{n, j}-(2 \pi k)^{2}\right)^{2}\right|}\right)\right)=O\left(\frac{\ln n}{n}\right) .
$$

Hence by (26) and (28) we obtain

$$
\Psi_{n, j}=u_{n, j} \varphi_{n}(x)+v_{n, j} \cos 2 \pi n x+O\left(\frac{\ln n}{n}\right) .
$$

Since $\Psi_{n, j}$ is normalized, we have

$$
\begin{aligned}
1= & \left\|\Psi_{n, j}\right\|^{2}=\left(\Psi_{n, j}, \Psi_{n, j}\right) \\
= & \left|u_{n, j}\right|^{2}\left\|\varphi_{n}\right\|^{2}+\left|v_{n, j}\right|^{2}\|\cos 2 \pi n x\|^{2} \\
& +u_{n, j} \bar{v}_{n, j}\left(\varphi_{n}, \cos 2 \pi n x\right)+v_{n, j} \bar{u}_{n, j}\left(\cos 2 \pi n x, \varphi_{n}\right)+O\left(\frac{\ln n}{n}\right) \\
= & \left(\frac{8}{3} \frac{|\beta|^{2}-\operatorname{Re} \beta+1}{|\beta-1|^{2}}\right)\left|u_{n, j}\right|^{2}+\frac{1}{2}\left|v_{n, j}\right|^{2}+O\left(\frac{\ln n}{n}\right),
\end{aligned}
$$


that is,

$$
a\left|u_{n, j}\right|^{2}+\frac{1}{2}\left|v_{n, j}\right|^{2}=1+O\left(\frac{\ln n}{n}\right),
$$

where

$$
a=\frac{8}{3} \frac{|\beta|^{2}-\operatorname{Re} \beta+1}{|\beta-1|^{2}} .
$$

Note that $a \neq 0$, since $|\beta|^{2}+1>|\beta|$ and by (30) we see that at least one of $u_{n, j}$ and $v_{n, j}$ is different from zero.

Now let us iterate (18). Using (22) in (18) we get

$$
\begin{aligned}
& \left(\lambda_{n, j}-(2 \pi n)^{2}\right)\left(\Psi_{n, j}, \sin 2 \pi n x\right) \\
& \quad=\sum_{n_{1}=0}^{\infty}\left[\left(q \varphi_{n_{1}}, \sin 2 \pi n x\right)\left(\Psi_{n, j}, \sin 2 \pi n_{1} x\right)+\left(q \cos 2 \pi n_{1} x, \sin 2 \pi n x\right)\left(\Psi_{n, j}, \varphi_{n_{1}}^{*}\right)\right] .
\end{aligned}
$$

Isolating the terms in the right-hand side of this equality containing the multiplicands $\left(\Psi_{n, j}, \sin 2 \pi n x\right)$ and $\left(\Psi_{n, j}, \varphi_{n}^{*}\right)$ (i.e., the case $\left.n_{1}=n\right)$, using (20) and (21) for the terms $\left(\Psi_{n, j}, \sin 2 \pi n_{1} x\right)$ and $\left(\Psi_{n, j}, \varphi_{n_{1}}^{*}\right)$, respectively (in the case $\left.n_{1} \neq n\right)$, we obtain

$$
\begin{aligned}
& {\left[\lambda_{n, j}-(2 \pi n)^{2}-\left(q \varphi_{n}, \sin 2 \pi n x\right)\right]\left(\Psi_{n, j}, \sin 2 \pi n x\right)-(q \cos 2 \pi n x, \sin 2 \pi n x)\left(\Psi_{n, j}, \varphi_{n}^{*}\right)} \\
& \quad=\sum_{\substack{n_{1}=0 \\
n_{1} \neq n}}^{\infty}\left[\left(q \varphi_{n_{1}}, \sin 2 \pi n x\right)\left(\Psi_{n, j}, \sin 2 \pi n_{1} x\right)+\left(q \cos 2 \pi n_{1} x, \sin 2 \pi n x\right)\left(\Psi_{n, j}, \varphi_{n_{1}}^{*}\right)\right] \\
& \quad=\sum_{n_{1}}\left[a_{1}\left(\lambda_{n, j}\right)\left(q(x) \Psi_{n, j}, \sin 2 \pi n_{1} x\right)+b_{1}\left(\lambda_{n, j}\right)\left(q(x) \Psi_{n, j}, \varphi_{n_{1}}^{*}\right)\right],
\end{aligned}
$$

where

$$
\begin{aligned}
& a_{1}\left(\lambda_{n, j}\right)=\frac{\left(q \varphi_{n_{1}}, \sin 2 \pi n x\right)}{\lambda_{n, j}-\left(2 \pi n_{1}\right)^{2}}+\frac{\gamma_{1} n_{1}\left(q \cos 2 \pi n_{1} x, \sin 2 \pi n x\right)}{\left(\lambda_{n, j}-\left(2 \pi n_{1}\right)^{2}\right)^{2}}, \\
& b_{1}\left(\lambda_{n, j}\right)=\frac{\left(q \cos 2 \pi n_{1} x, \sin 2 \pi n x\right)}{\lambda_{n, j}-\left(2 \pi n_{1}\right)^{2}} .
\end{aligned}
$$

Using (22) and (23) for the terms $\left(q \Psi_{n, j}, \sin 2 \pi n_{1} x\right)$ and $\left(q \Psi_{n, j}, \varphi_{n_{1}}^{*}\right)$ of the last summation we obtain

$$
\begin{aligned}
& {\left[\lambda_{n, j}-(2 \pi n)^{2}-\left(q \varphi_{n}, \sin 2 \pi n x\right)\right]\left(\Psi_{n, j}, \sin 2 \pi n x\right)-(q \cos 2 \pi n x, \sin 2 \pi n x)\left(\Psi_{n, j}, \varphi_{n}^{*}\right)} \\
& =\sum_{n_{1}}\left[a_{1}\left(\lambda_{n, j}\right)\left(q \Psi_{n, j}, \sin 2 \pi n_{1} x\right)+b_{1}\left(\lambda_{n, j}\right)\left(q \Psi_{n, j}, \varphi_{n_{1}}^{*}\right)\right] \\
& =\sum_{n_{1}} a_{1}\left(\sum _ { n _ { 2 } = 0 } ^ { \infty } \left[\left(q \varphi_{n_{2}}, \sin 2 \pi n_{1} x\right)\left(\Psi_{n, j}, \sin 2 \pi n_{2} x\right)\right.\right. \\
& \left.\left.\quad+\left(q \cos 2 \pi n_{2} x, \sin 2 \pi n_{1} x\right)\left(\Psi_{n, j}, \varphi_{n_{2}}^{*}\right)\right]\right)
\end{aligned}
$$




$$
\begin{aligned}
& +\sum_{n_{1}} b_{1}\left(\sum _ { n _ { 2 } = 0 } ^ { \infty } \left[\left(q \varphi_{n_{2}}, \varphi_{n_{1}}^{*}\right)\left(\Psi_{n, j}, \sin 2 \pi n_{2} x\right)\right.\right. \\
& \left.\left.+\left(q \cos 2 \pi n_{2} x, \varphi_{n_{1}}^{*}\right)\left(\Psi_{n, j}, \varphi_{n_{2}}^{*}\right)\right]\right) .
\end{aligned}
$$

Now isolating the terms for $n_{2}=n$ we get

$$
\begin{aligned}
& {\left[\lambda_{n, j}-(2 \pi n)^{2}-\left(q \varphi_{n}, \sin 2 \pi n x\right)\right]\left(\Psi_{n, j}, \sin 2 \pi n x\right)-(q \cos 2 \pi n x, \sin 2 \pi n x)\left(\Psi_{n, j}, \varphi_{n}^{*}\right)} \\
& =\sum_{n_{1}}\left[a_{1}\left(q \varphi_{n}, \sin 2 \pi n_{1} x\right)+b_{1}\left(q \varphi_{n}, \varphi_{n_{1}}^{*}\right)\right]\left(\Psi_{n, j}, \sin 2 \pi n x\right) \\
& \quad+\sum_{n_{1}}\left[a_{1}\left(q \cos 2 \pi n x, \sin 2 \pi n_{1} x\right)+b_{1}\left(q \cos 2 \pi n x, \varphi_{n_{1}}^{*}\right)\right]\left(\Psi_{n, j}, \varphi_{n}^{*}(x)\right) \\
& =\sum_{n_{1}, n_{2}}\left(\left[a_{1}\left(q \varphi_{n_{2}}, \sin 2 \pi n_{1} x\right)+b_{1}\left(q \varphi_{n_{2}}, \varphi_{n_{1}}^{*}\right)\right]\left(\Psi_{n, j}, \sin 2 \pi n_{2} x\right)\right) \\
& \quad+\sum_{n_{1}, n_{2}}\left[a_{1}\left(q \cos 2 \pi n_{2} x, \sin 2 \pi n_{1} x\right)+b_{1}\left(q \cos 2 \pi n_{2} x, \varphi_{n_{1}}^{*}\right)\right]\left(\Psi_{n, j}, \varphi_{n_{2}}^{*}\right) .
\end{aligned}
$$

Here and below the summations are taken under the conditions $n_{i} \neq n$ and $n_{i}=0,1, \ldots$ for $i=1,2, \ldots$ Introduce the notations

$$
\begin{aligned}
& C_{1}=: a_{1}, \quad M_{1}=: b_{1}, \\
& C_{k+1}=: C_{k} a_{k+1}+M_{k} A_{k+1}, \quad M_{k+1}=: C_{k} b_{k+1}+M_{k} B_{k+1} ; \quad k=1,2, \ldots,
\end{aligned}
$$

where

$$
\begin{aligned}
& a_{k+1}=a_{k+1}\left(\lambda_{n, j}\right)=\frac{\left(q \varphi_{n_{k+1}}, \sin 2 \pi n_{k} x\right)}{\lambda_{n, j}-\left(2 \pi n_{k+1}\right)^{2}}+\frac{\gamma_{1} n_{k+1}\left(q \cos 2 \pi n_{k+1} x, \sin 2 \pi n_{k} x\right)}{\left(\lambda_{n, j}-\left(2 \pi n_{k+1}\right)^{2}\right)^{2}}, \\
& b_{k+1}=b_{k+1}\left(\lambda_{n, j}\right)=\frac{\left(q \cos 2 \pi n_{k+1} x, \sin 2 \pi n_{k} x\right)}{\lambda_{n, j}-\left(2 \pi n_{k+1}\right)^{2}}, \\
& A_{k+1}=A_{k+1}\left(\lambda_{n, j}\right)=\frac{\left(q \varphi_{n_{k+1}}, \varphi_{n_{k}}^{*}\right)}{\lambda_{n, j}-\left(2 \pi n_{k+1}\right)^{2}}+\frac{\gamma_{1} n_{k+1}\left(q \cos 2 \pi n_{k+1} x, \varphi_{n_{k}}^{*}\right)}{\left(\lambda_{n, j}-\left(2 \pi n_{k+1}\right)^{2}\right)^{2}}, \\
& B_{k+1}=B_{k+1}\left(\lambda_{n, j}\right)=\frac{\left(q \cos 2 \pi n_{k+1} x, \varphi_{n_{k}}^{*}\right)}{\lambda_{n, j}-\left(2 \pi n_{k+1}\right)^{2}} .
\end{aligned}
$$

Using these notations and repeating this iteration $k$ times we get

$$
\begin{gathered}
{\left[\lambda_{n, j}-(2 \pi n)^{2}-\left(q \varphi_{n}, \sin 2 \pi n x\right)-\widetilde{A}_{k}\left(\lambda_{n, j}\right)\right]\left(\Psi_{n, j}, \sin 2 \pi n x\right)} \\
\quad=\left[(q \cos 2 \pi n x, \sin 2 \pi n x)+\widetilde{B}_{k}\left(\lambda_{n, j}\right)\right]\left(\Psi_{n, j}, \varphi_{n}^{*}(x)\right)+R_{k},
\end{gathered}
$$

where

$$
\begin{aligned}
& \widetilde{A}_{k}\left(\lambda_{n, j}\right)=\sum_{m=1}^{k} \alpha_{m}\left(\lambda_{n, j}\right), \quad \widetilde{B}_{k}\left(\lambda_{n, j}\right)=\sum_{m=1}^{k} \beta_{m}\left(\lambda_{n, j}\right), \\
& \alpha_{k}\left(\lambda_{n, j}\right)=\sum_{n_{1}, \ldots, n_{k}}\left[C_{k}\left(q \varphi_{n}, \sin 2 \pi n_{k} x\right)+M_{k}\left(q \varphi_{n}, \varphi_{n_{k}}^{*}\right)\right]
\end{aligned}
$$




$$
\begin{aligned}
& \beta_{k}\left(\lambda_{n, j}\right)=\sum_{n_{1}, \ldots, n_{k}}\left[C_{k}\left(q \cos 2 \pi n x, \sin 2 \pi n_{k} x\right)+M_{k}\left(q \cos 2 \pi n x, \varphi_{n_{k}}^{*}\right)\right], \\
& R_{k}=\sum_{n_{1}, \ldots, n_{k+1}}\left\{C_{k+1}\left(q \Psi_{n, j}, \sin 2 \pi n_{k+1} x\right)+M_{k+1}\left(q \Psi_{n, j}, \varphi_{n_{k+1}}^{*}\right)\right\} .
\end{aligned}
$$

It follows from (10), (11), (24), and (25) that

$$
\begin{aligned}
& \alpha_{k}(\lambda)=O\left(\left(\frac{\ln |n|}{n}\right)^{k}\right), \quad \beta_{k}(\lambda)=O\left(\left(\frac{\ln |n|}{n}\right)^{k}\right), \\
& R_{k}(\lambda)=O\left(\left(\frac{\ln |n|}{n}\right)^{k+1}\right)
\end{aligned}
$$

for $\lambda=\lambda_{n, j}$ and for all $\lambda \in U(n)$, where $U(n)=\left\{\lambda:\left|\lambda-(2 \pi n)^{2}\right| \leq n\right\}$.

Therefore letting $k$ tend to infinity, we obtain

$$
\left[\lambda_{n, j}-(2 \pi n)^{2}-Q_{n}-A\left(\lambda_{n, j}\right)\right] u_{n, j}=\left[P_{n}+B\left(\lambda_{n, j}\right)\right] v_{n, j},
$$

where

$$
\begin{aligned}
& P_{n}=(q \cos 2 \pi n x, \sin 2 \pi n x), \quad Q_{n}=\left(q \varphi_{n}, \sin 2 \pi n x\right), \\
& A(\lambda)=\sum_{m=1}^{\infty} \alpha_{m}(\lambda), \quad B(\lambda)=\sum_{m=1}^{\infty} \beta_{m}(\lambda)
\end{aligned}
$$

and by (32) we have

$$
A(\lambda)=O\left(\frac{\ln |n|}{n}\right), \quad B(\lambda)=O\left(\frac{\ln |n|}{n}\right)
$$

for $\lambda=\lambda_{n, j}$ and for all $\lambda \in U(n)$.

Thus iterating (18) we obtain (31). Now iterating (19) instead of (18), using (23) and (22), and arguing as in the previous iteration, we get

$$
\left[\lambda_{n, j}-(2 \pi n)^{2}-P_{n}^{*}-A_{k}^{\prime}\left(\lambda_{n, j}\right)\right] v_{n, j}=\left[\gamma_{1} n+Q_{n}^{*}+B_{k}^{\prime}\left(\lambda_{n, j}\right)\right] u_{n, j}+R_{k}^{\prime},
$$

where

$$
\begin{aligned}
& P_{n}^{*}=\left(q \cos 2 \pi n x, \varphi_{n}^{*}\right), \quad Q_{n}^{*}=\left(q \varphi_{n}, \varphi_{n}^{*}\right), \\
& A_{k}^{\prime}\left(\lambda_{n, j}\right)=\sum_{m=1}^{k} \alpha_{m}^{\prime}\left(\lambda_{n, j}\right), \quad B_{k}^{\prime}\left(\lambda_{n, j}\right)=\sum_{m=1}^{k} \beta_{m}^{\prime}\left(\lambda_{n, j}\right), \\
& \alpha_{k}^{\prime}\left(\lambda_{n, j}\right)=\sum_{n_{1}, \ldots, n_{k}}\left[\widetilde{C}_{k}\left(q \cos 2 \pi n x, \sin 2 \pi n_{k} x\right)+\widetilde{M}_{k}\left(q \cos 2 \pi n x, \varphi_{n_{k}}^{*}\right)\right], \\
& \beta_{k}^{\prime}\left(\lambda_{n, j}\right)=\sum_{n_{1}, \ldots, n_{k}}\left[\widetilde{C}_{k}\left(q \varphi_{n}, \sin 2 \pi n_{k} x\right)+\widetilde{M}_{k}\left(q \varphi_{n}, \varphi_{n_{k}}^{*}\right)\right], \\
& R_{k}^{\prime}=\sum_{n_{1}, \ldots, n_{k+1}}\left\{\widetilde{C}_{k+1}\left(q \Psi_{n, j}, \sin 2 \pi n_{k+1} x\right)+\widetilde{M}_{k+1}\left(q \Psi_{n, j}, \varphi_{n_{k+1}}^{*}\right)\right\},
\end{aligned}
$$




$$
\begin{aligned}
& \widetilde{C}_{k+1}=\widetilde{C}_{k} a_{k+1}+\widetilde{M}_{k} A_{k+1}, \quad \widetilde{M}_{k+1}=\widetilde{C}_{k} b_{k+1}+\widetilde{M}_{k} B_{k+1} ; \quad k=1,2, \ldots, \\
& \widetilde{C}_{1}=A_{1}\left(\lambda_{n, j}\right)=\frac{\left(q \varphi_{n_{1}}, \varphi_{n}^{*}\right)}{\lambda_{n, j}-\left(2 \pi n_{1}\right)^{2}}+\frac{\gamma_{1} n_{1}\left(q \cos 2 \pi n_{1} x, \varphi_{n}^{*}\right)}{\left(\lambda_{n, j}-\left(2 \pi n_{1}\right)^{2}\right)^{2}}, \\
& \widetilde{M}_{1}=B_{1}\left(\lambda_{n, j}\right)=\frac{\left(q \cos 2 \pi n_{1} x, \varphi_{n}^{*}\right)}{\lambda_{n, j}-\left(2 \pi n_{1}\right)^{2}} .
\end{aligned}
$$

Similar to (32) one can verify that

$$
\begin{aligned}
& \alpha_{k}^{\prime}(\lambda)=O\left(\left(\frac{\ln |n|}{n}\right)^{k}\right), \quad \beta_{k}^{\prime}(\lambda)=O\left(\left(\frac{\ln |n|}{n}\right)^{k}\right), \\
& R_{k}^{\prime}(\lambda)=O\left(\left(\frac{\ln |n|}{n}\right)^{k+1}\right)
\end{aligned}
$$

for $\lambda=\lambda_{n, j}$ and for all $\lambda \in U(n)$. Now letting $k$ tend to infinity in (35), we obtain

$$
\left[\lambda_{n, j}-(2 \pi n)^{2}-P_{n}^{*}-A^{\prime}\left(\lambda_{n, j}\right)\right] v_{n, j}=\left[\gamma_{1} n+Q_{n}^{*}+B^{\prime}\left(\lambda_{n, j}\right)\right] u_{n, j}
$$

where

$$
A^{\prime}\left(\lambda_{n, j}\right)=\sum_{m=1}^{\infty} \alpha_{m}^{\prime}\left(\lambda_{n, j}\right), \quad B^{\prime}\left(\lambda_{n, j}\right)=\sum_{m=1}^{\infty} \beta_{m}^{\prime}\left(\lambda_{n, j}\right)
$$

and by (37) we have

$$
A^{\prime}(\lambda)=O\left(\frac{\ln |n|}{n}\right), \quad B^{\prime}(\lambda)=O\left(\frac{\ln |n|}{n}\right)
$$

for $\lambda=\lambda_{n, j}$ and for all $\lambda \in U(n)$.

To get the main results of this paper we use the following system of equations, obtained above, with respect to $u_{n, j}$ and $v_{n, j}$,

$$
\begin{aligned}
& {\left[\lambda_{n, j}-(2 \pi n)^{2}-Q_{n}-A\left(\lambda_{n, j}\right)\right] u_{n, j}=\left[P_{n}+B\left(\lambda_{n, j}\right)\right] v_{n, j},} \\
& {\left[\lambda_{n, j}-(2 \pi n)^{2}-P_{n}^{*}-A^{\prime}\left(\lambda_{n, j}\right)\right] v_{n, j}=\left[\gamma_{1} n+Q_{n}^{*}+B^{\prime}\left(\lambda_{n, j}\right)\right] u_{n, j},}
\end{aligned}
$$

where

$$
\begin{aligned}
Q_{n}= & -\frac{2(\beta+1)}{\beta-1} \int_{0}^{1} x q(x) d x+\frac{2(\beta+1)}{\beta-1}(x q(x), \cos 4 \pi n x) \\
& -\frac{2 \beta}{\beta-1}(q(x), \cos 4 \pi n x) \\
= & -\frac{2(\beta+1)}{\beta-1} \int_{0}^{1} x q(x) d x+o(1), \\
P_{n}^{*}= & \frac{2(\beta+1)}{\beta-1} \int_{0}^{1} x q(x) d x+\frac{2(\beta+1)}{\beta-1}(x q(x), \cos 4 \pi n x)-\frac{2}{\beta-1}(q(x), \cos 4 \pi n x) \\
= & \frac{2(\beta+1)}{\beta-1} \int_{0}^{1} x q(x) d x+o(1),
\end{aligned}
$$




$$
\begin{aligned}
& P_{n}=\frac{1}{2}(q, \sin 4 \pi n x)=o(1), \\
& Q_{n}^{*}=8\left(\frac{\beta_{1}+1}{\beta_{1}-1}\right)^{2} \int_{0}^{1} q(x)\left(\frac{\beta_{1}}{1+\beta_{1}}-x\right)\left(x-\frac{1}{1+\beta_{1}}\right) \sin 4 \pi n x d x=o(1)
\end{aligned}
$$

(see (33) and (36)). Note that (39), (40) with (34), (38) give

$$
\begin{aligned}
& {\left[\lambda_{n, j}-(2 \pi n)^{2}-Q_{n}+O\left(\frac{\ln |n|}{n}\right)\right] u_{n, j}=\left[P_{n}+O\left(\frac{\ln |n|}{n}\right)\right] v_{n, j},} \\
& {\left[\lambda_{n, j}-(2 \pi n)^{2}-P_{n}^{*}+O\left(\frac{\ln |n|}{n}\right)\right] v_{n, j}=\left[\gamma_{1} n+Q_{n}^{*}+O\left(\frac{\ln |n|}{n}\right)\right] u_{n, j} .}
\end{aligned}
$$

Introduce the notations

$$
\begin{aligned}
& c_{n}=(q, \cos 2 \pi n x), \quad s_{n}=(q, \sin 2 \pi n x), \\
& c_{n, 1}=(x q, \cos 2 \pi n x), \quad s_{n, 1}=(x q, \sin 2 \pi n x), \\
& c_{n, 2}=\left(x^{2} q, \cos 2 \pi n x\right), \quad s_{n, 2}=\left(x^{2} q, \sin 2 \pi n x\right) .
\end{aligned}
$$

Then, by (41)-(46) and (49) we have

$$
\begin{aligned}
& Q_{n}=-\frac{2(\beta+1)}{\beta-1} \int_{0}^{1} x q(x) d x+\frac{2(\beta+1)}{\beta-1} c_{2 n, 1}-\frac{2 \beta}{\beta-1} c_{2 n}, \\
& P_{n}^{*}=\frac{2(\beta+1)}{\beta-1} \int_{0}^{1} x q(x) d x+\frac{2(\beta+1)}{\beta-1} c_{2 n, 1}-\frac{2}{\beta-1} c_{2 n}, \\
& P_{n}=\frac{1}{2} s_{2 n}, \\
& Q_{n}^{*}=-8\left(\frac{\beta+1}{\beta-1}\right)^{2} s_{2 n, 2}+8\left(\frac{\beta+1}{\beta-1}\right)^{2} s_{2 n, 1}-\frac{8 \beta}{(\beta-1)^{2}} s_{2 n} .
\end{aligned}
$$

Theorem 1 The following statements hold:

(a) Any eigenfunction $\Psi_{n, j}$ of $T_{1}$ corresponding to the eigenvalue $\lambda_{n, j}$ defined in (10) satisfies

$$
\Psi_{n, j}=\sqrt{2} \cos 2 \pi n x+O\left(n^{-1 / 2}\right) .
$$

Moreover, there exists $N$ such that for all $n>N$ the geometric multiplicity of the eigenvalue $\lambda_{n, j}$ is 1 .

(b) A complex number $\lambda \in U(n)$, where $U(n)$ is defined in (32), is an eigenvalue of $T_{1}$ if and only if it is a root of the equation

$$
\begin{aligned}
& {\left[\lambda-(2 \pi n)^{2}-Q_{n}-A(\lambda)\right]\left[\lambda-(2 \pi n)^{2}-P_{n}^{*}-A^{\prime}(\lambda)\right]} \\
& \quad-\left[P_{n}+B(\lambda)\right]\left[\gamma_{1} n+Q_{n}^{*}+B^{\prime}(\lambda)\right]=0 .
\end{aligned}
$$

Moreover, $\lambda \in U(n)$ is a double eigenvalue of $T_{1}$ if and only if it is a double root of (55).

Proof (a) By (10) the left-hand side of (48) is $O\left(n^{1 / 2}\right)$, which implies that $u_{n, j}=O\left(n^{-1 / 2}\right)$. Therefore from (29) we obtain (54). Now suppose that there are two linearly independent 
eigenfunctions corresponding to $\lambda_{n, j}$. Then there exists an eigenfunction satisfying

$$
\Psi_{n, j}=\sqrt{2} \sin 2 \pi n x+o(1)
$$

which contradicts (54).

(b) First we prove that the large eigenvalues $\lambda_{n, j}$ are the roots of (55). It follows from (54), (27), and (15) that $v_{n, j} \neq 0$. If $u_{n, j} \neq 0$ then multiplying (39) and (40) side by side and then canceling $v_{n, j} u_{n, j}$ we obtain (55). If $u_{n, j}=0$ then by (39) and (40) we have $P_{n}+B\left(\lambda_{n, j}\right)=0$ and $\lambda_{n, j}-(2 \pi n)^{2}-P_{n}^{*}-A^{\prime}\left(\lambda_{n, j}\right)=0$, which means that (55) holds. Thus in any case $\lambda_{n, j}$ is a root of (55).

Now we prove that the roots of (55) lying in $U(n)$ are the eigenvalues of $T_{1}$. Let $F(\lambda)$ be the left-hand side of (55), which can be written as

$$
\begin{aligned}
F(\lambda)= & \left(\lambda-(2 \pi n)^{2}\right)^{2}-\left(Q_{n}+A(\lambda)+P_{n}^{*}+A^{\prime}(\lambda)\right)\left(\lambda-(2 \pi n)^{2}\right) \\
& +\left(Q_{n}+A(\lambda)\right)\left(P_{n}^{*}+A^{\prime}(\lambda)\right)-\left(P_{n}+B(\lambda)\right)\left(\gamma_{1} n+Q_{n}^{*}+B^{\prime}(\lambda)\right)
\end{aligned}
$$

and

$$
G(\lambda)=\left(\lambda-(2 \pi n)^{2}\right)^{2}
$$

Using (34) and (38), one can easily verify that the inequality

$$
|F(\lambda)-G(\lambda)|<|G(\lambda)|
$$

holds for all $\lambda$ from the boundary of $U(n)$. Since the function $G(\lambda)$ has two roots in the set $U(n)$, by the Rouche theorem we find that $F(\lambda)$ has two roots in the same set. Thus $T_{1}$ has two eigenvalues (counting with multiplicities) lying in $U(n)$ that are the roots of (55). On the other hand, (55) has preciously two roots (counting with multiplicities) in $U(n)$. Therefore $\lambda \in U(n)$ is an eigenvalue of $T_{1}$ if and only if (55) holds.

If $\lambda \in U(n)$ is a double eigenvalue of $T_{1}$ then it has no other eigenvalues in $U(n)$ and hence (55) has no other roots. This implies that $\lambda$ is a double root of (55). By the same way one can prove that if $\lambda$ is a double root of (55) then it is a double eigenvalue of $T_{1}$.

Let us consider (55) in detail. By (56) we have

$$
F(\lambda)=0 .
$$

If we substitute $t=: \lambda-(2 \pi n)^{2}$ in (57), then it becomes

$$
\begin{aligned}
t^{2} & -\left(Q_{n}+A(\lambda)+P_{n}^{*}+A^{\prime}(\lambda)\right) t+\left(Q_{n}+A(\lambda)\right)\left(P_{n}^{*}+A^{\prime}(\lambda)\right) \\
& -\left(P_{n}+B(\lambda)\right)\left(\gamma_{1} n+Q_{n}^{*}+B^{\prime}(\lambda)\right)=0 .
\end{aligned}
$$

The solutions of (58) are

$$
t_{1,2}=\frac{\left(Q_{n}+P_{n}^{*}+A+A^{\prime}\right) \pm \sqrt{\Delta(\lambda)}}{2},
$$


where

$$
\Delta(\lambda)=\left(Q_{n}+P_{n}^{*}+A+A^{\prime}\right)^{2}-4\left(Q_{n}+A\right)\left(P_{n}^{*}+A^{\prime}\right)+4\left(P_{n}+B\right)\left(\gamma_{1} n+Q_{n}^{*}+B^{\prime}\right),
$$

which can be written in the form

$$
\Delta(\lambda)=\left(Q_{n}-P_{n}^{*}+A-A^{\prime}\right)^{2}+4\left(P_{n}+B\right)\left(\gamma_{1} n+Q_{n}^{*}+B^{\prime}\right)
$$

and, as we shall see below, $\sqrt{\Delta(\lambda)}$ can be defined as analytic function on $U(n)$. Clearly the eigenvalue $\lambda_{n, j}$ is a root either of the equation

$$
\lambda=(2 \pi n)^{2}+\frac{1}{2}\left[\left(Q_{n}+P_{n}^{*}+A+A^{\prime}\right)-\sqrt{\Delta(\lambda)}\right]
$$

or of the equation

$$
\lambda=(2 \pi n)^{2}+\frac{1}{2}\left[\left(Q_{n}+P_{n}^{*}+A+A^{\prime}\right)+\sqrt{\Delta(\lambda)}\right] .
$$

Now let us examine $\Delta(\lambda)$ and $\sqrt{\Delta(\lambda)}$ in detail. If (8) holds then one can readily see from (34), (38), (50)-(53), and (59) that

$$
\Delta(\lambda)=2 \gamma_{1} n s_{2 n}(1+o(1))
$$

for $\lambda \in U(n)$. By (62) there exists an appropriate choice of branch of $\sqrt{\Delta(\lambda)}$ (depending on $n$ ) which is analytic on $U(n)$. Taking into account (62), (34), (38), (50), and (51), we see that (60) and (61) have the form

$$
\begin{aligned}
& \lambda=(2 \pi n)^{2}-\frac{\sqrt{2 \gamma_{1}}}{2} \sqrt{n s_{2 n}}(1+o(1)), \\
& \lambda=(2 \pi n)^{2}+\frac{\sqrt{2 \gamma_{1}}}{2} \sqrt{n s_{2 n}}(1+o(1)) .
\end{aligned}
$$

Theorem 2 If (8) holds, then the large eigenvalues $\lambda_{n, j}$ are simple and satisfy the following asymptotic formulas:

$$
\lambda_{n, j}=(2 \pi n)^{2}+(-1)^{j} \frac{\sqrt{2 \gamma_{1}}}{2} \sqrt{n s_{2 n}}(1+o(1))
$$

for $j=1,2$. Moreover, if there exists a sequence $\left\{n_{k}\right\}$ such that (8) holds when $n$ is replaced by $n_{k}$, then the root functions of $T_{1}$ do not form a Riesz basis.

Proof To prove that the large eigenvalues $\lambda_{n, j}$ are simple let us show that one of the eigenvalues, say $\lambda_{n, 1}$ satisfies (65) for $j=1$ and the other $\lambda_{n, 2}$ satisfies (65) for $j=2$. Let us prove that each of (60) and (61) has a unique root in $U(n)$ by proving that

$$
(2 \pi n)^{2}+\frac{1}{2}\left[\left(Q_{n}+P_{n}^{*}+A+A^{\prime}\right) \pm \sqrt{\Delta(\lambda)}\right]
$$

is a contraction mapping. For this we show that there exist positive real numbers $K_{1}, K_{2}$, 
$K_{3}$ such that

$$
\begin{aligned}
& |A(\lambda)-A(\mu)|<K_{1}|\lambda-\mu|, \quad\left|A^{\prime}(\lambda)-A^{\prime}(\mu)\right|<K_{2}|\lambda-\mu|, \\
& |\sqrt{\Delta(\lambda)}-\sqrt{\Delta(\mu)}|<K_{3}|\lambda-\mu|
\end{aligned}
$$

for $\lambda, \mu \in U(n)$, where $K_{1}+K_{2}+K_{3}<1$. The proof of (66) is similar to the proof of (56) of the paper [26].

Now let us prove (67). By (62) and (8) we have

$$
(\sqrt{\Delta(\lambda)})^{-1}=o(1)
$$

for $\lambda \in U(n)$. On the other hand arguing as in the proof of (56) of the paper [26] we get

$$
\frac{d}{d \lambda} \Delta(\lambda)=O(1)
$$

Hence for the large values of $n$ we have

$$
\frac{d}{d \lambda} \sqrt{\Delta(\lambda)}=\frac{\frac{d}{d \lambda} \Delta(\lambda)}{2 \sqrt{\Delta(\lambda)}}=o(1)
$$

for $\lambda \in U(n)$. Thus by the fixed point theorem, each of (60) and (61) has a unique root $\lambda_{1}$ and $\lambda_{2}$ in $U(n)$ respectively. Clearly by (63) and (64), we have $\lambda_{1} \neq \lambda_{2}$ which implies that (55) has two simple roots in $U(n)$. Therefore by Theorem $1(\mathrm{~b}), \lambda_{1}$ and $\lambda_{2}$ are the eigenvalues of $T_{1}$ lying in $U(n)$, that is, they are $\lambda_{n, 1}$ and $\lambda_{n, 2}$, which proves the simplicity of the large eigenvalues and the validity of (65).

If there exists a sequence $\left\{n_{k}\right\}$ such that (8) holds when $n$ is replaced by $n_{k}$, then by Theorem 1(a)

$$
\left(\Psi_{n_{k}, 1}, \Psi_{n_{k}, 2}\right)=1+O\left(n_{k}^{-1 / 2}\right)
$$

Now it follows from the theorems of $[4,5]$ (see also Lemma 3 of [18]) that the root functions of $T_{1}$ do not form a Riesz basis.

Now let us consider the operators $T_{2}, T_{3}$, and $T_{4}$. First we consider the operator $T_{3}$.

It is well known that (see (47a) and (47b) on page 65 of [24]) the eigenvalues of the operators $T_{3}(q)$ consist of the sequences $\left\{\lambda_{n, 1,3}\right\},\left\{\lambda_{n, 2,3}\right\}$ satisfying $(10)$ when $\lambda_{n, j}$ is replaced by $\lambda_{n, j, 3}$. The eigenvalues, eigenfunctions and associated functions of $T_{3}(0)$ are

$$
\begin{aligned}
& \lambda_{n, 3}=(2 \pi n)^{2} ; \quad n=0,1,2, \ldots, \\
& y_{0,3}(x)=x-\frac{\alpha}{1+\alpha}, \quad y_{n, 3}(x)=\sin 2 \pi n x ; \quad n=1,2, \ldots, \\
& \phi_{n, 3}(x)=\left(x-\frac{\alpha}{1+\alpha}\right) \frac{\cos 2 \pi n x}{4 \pi n} ; \quad n=1,2, \ldots,
\end{aligned}
$$

respectively. The biorthogonal systems analogous to (16) and (17) are

$$
\left\{\cos 2 \pi n x, \frac{4(1+\bar{\alpha})}{1-\bar{\alpha}}\left(\frac{1}{1+\bar{\alpha}}-x\right) \sin 2 \pi n x\right\}_{n=0}^{\infty},
$$




$$
\left\{\sin 2 \pi n x, \frac{4(1+\alpha)}{1-\alpha}\left(x-\frac{\alpha}{1+\alpha}\right) \cos 2 \pi n x\right\}_{n=0}^{\infty},
$$

respectively.

Analogous formulas to (18) and (19) are

$$
\begin{aligned}
& \left(\lambda_{N, j, 3}-(2 \pi n)^{2}\right)\left(\Psi_{N, j, 3}, \cos 2 \pi n x\right)=\left(q \Psi_{N, j, 3}, \cos 2 \pi n x\right), \\
& \left(\lambda_{N, j, 3}-(2 \pi n)^{2}\right)\left(\Psi_{N, j, 3}, \varphi_{n, 3}^{*}\right)-\gamma_{3} n\left(\Psi_{N, j, 3}, \cos 2 \pi n x\right)=\left(q(x) \Psi_{N, j, 3}, \varphi_{n, 3}^{*}\right),
\end{aligned}
$$

respectively, where

$$
\gamma_{3}=\frac{16 \pi(1+\alpha)}{1-\alpha}
$$

Instead of (16)-(19) using (68)-(71) and arguing as in the proofs of Theorem 1 and Theorem 2 we obtain the following results for $T_{3}$.

Theorem 3 If $(8)$ holds, then the large eigenvalues $\lambda_{n, j, 3}$ are simple and satisfy the following asymptotic formulas:

$$
\lambda_{n, j, 3}=(2 \pi n)^{2}+(-1)^{j} \frac{\sqrt{2 \gamma_{3}}}{2} \sqrt{n s_{2 n}}(1+o(1))
$$

for $j=1,2$. The eigenfunctions $\Psi_{n, j, 3}$ corresponding to $\lambda_{n, j, 3}$ obey

$$
\Psi_{n, j, 3}=\sqrt{2} \sin 2 \pi n x+O\left(n^{-1 / 2}\right) .
$$

Moreover, if there exists a sequence $\left\{n_{k}\right\}$ such that (8) holds when $n$ is replaced by $n_{k}$, then the root functions of $T_{3}$ do not form a Riesz basis.

Now let us consider the operator $T_{2}$. It is well known that (see (47a) and (47b) on page 65 of [24]) the eigenvalues of the operators $T_{2}(q)$ consist of the sequences $\left\{\lambda_{n, 1,2}\right\},\left\{\lambda_{n, 2,2}\right\}$ satisfying

$$
\lambda_{n, j, 2}=(2 n \pi+\pi)^{2}+O\left(n^{1 / 2}\right)
$$

for $j=1,2$. The eigenvalues, eigenfunctions, and associated functions of $T_{2}(0)$ are

$$
\begin{aligned}
& (\pi+2 \pi n)^{2}, \quad y_{n, 2}(x)=\cos (2 n+1) \pi x, \\
& \phi_{n, 2}(x)=\left(\frac{\beta}{\beta-1}-x\right) \frac{\sin (2 n+1) \pi x}{2(2 n+1) \pi}
\end{aligned}
$$

for $n=0,1,2, \ldots$, respectively. The biorthogonal systems analogous to (16) and (17) are

$$
\begin{aligned}
& \left\{\sin (2 n+1) \pi x, \frac{4(\bar{\beta}-1)}{\bar{\beta}+1}\left(x+\frac{1}{\bar{\beta}-1}\right) \cos (2 n+1) \pi x\right\}_{n=0}^{\infty}, \\
& \left\{\cos (2 n+1) \pi x, \frac{4(\beta-1)}{\beta+1}\left(\frac{\beta}{\beta-1}-x\right) \sin (2 n+1) \pi x\right\}_{n=0}^{\infty},
\end{aligned}
$$

respectively. 
Analogous formulas to (18) and (19) are

$$
\begin{aligned}
& \left(\lambda_{N, j, 2}-((2 n+1) \pi)^{2}\right)\left(\Psi_{N, j, 2}, \sin (2 n+1) \pi x\right)=\left(q \Psi_{N, j, 2}, \sin (2 n+1) \pi x\right) \\
& \left(\lambda_{N, j, 2}-((2 n+1) \pi)^{2}\right)\left(\Psi_{N, j, 2}, \varphi_{n, 2}^{*}\right)-(2 n+1) \gamma_{2}\left(\Psi_{N, j, 2}, \sin (2 n+1) \pi x\right) \\
& \quad=\left(q \Psi_{N, j, 2}, \varphi_{n, 2}^{*}\right)
\end{aligned}
$$

respectively, where

$$
\gamma_{2}=\frac{8 \pi(\beta-1)}{\beta+1}
$$

Instead of (16)-(19) using (73)-(76) and arguing as in the proofs of Theorem 1 and Theorem 2 we obtain the following results for $T_{2}$.

Theorem 4 If (9) holds, then the large eigenvalues $\lambda_{n, j, 2}$ are simple and satisfy the following asymptotic formulas:

$$
\lambda_{n, j, 2}=((2 n+1) \pi)^{2}+(-1)^{j} \frac{\sqrt{2 \gamma_{2}}}{2} \sqrt{(2 n+1) s_{2 n+1}}(1+o(1))
$$

for $j=1,2$. The eigenfunctions $\Psi_{n, j, 2}$ corresponding to $\lambda_{n, j, 2}$ obey

$$
\Psi_{n, j, 2}=\sqrt{2} \cos (2 n+1) \pi x+O\left(n^{-1 / 2}\right) .
$$

Moreover, if there exists a sequence $\left\{n_{k}\right\}$ such that (9) holds when $n$ is replaced by $n_{k}$, then the root functions of $T_{2}$ do not form a Riesz basis.

Lastly we consider the operator $T_{4}$. It is well known that (see (47a) and (47b) on page 65 of [24]) the eigenvalues of the operators $T_{4}(q)$ consist of the sequences $\left\{\lambda_{n, 1,4}\right\},\left\{\lambda_{n, 2,4}\right\}$ satisfying (72) when $\lambda_{n, j, 2}$ is replaced by $\lambda_{n, j, 4}$. The eigenvalues, eigenfunctions, and associated functions of $T_{4}(0)$ are

$$
\begin{aligned}
& \lambda_{n, 4}=(\pi+2 \pi n)^{2}, \quad y_{n, 4}(x)=\sin (2 n+1) \pi x, \\
& \phi_{n, 4}(x)=\left(\frac{\alpha}{1-\alpha}+x\right) \frac{\cos (2 n+1) \pi x}{2(2 n+1) \pi}
\end{aligned}
$$

for $n=0,1,2, \ldots$, respectively. The biorthogonal systems analogous to (16) and (17) are

$$
\begin{aligned}
& \left\{\cos (2 n+1) \pi x, \frac{4(1-\bar{\alpha})}{1+\bar{\alpha}}\left(\frac{1}{1-\bar{\alpha}}-x\right) \sin (2 n+1) \pi x\right\}_{n=0}^{\infty}, \\
& \left\{\sin (2 n+1) \pi x, \frac{4(1-\alpha)}{1+\alpha}\left(\frac{\alpha}{1-\alpha}+x\right) \cos (2 n+1) \pi x\right\}_{n=0}^{\infty},
\end{aligned}
$$

respectively.

Analogous formulas to (18) and (19) are

$$
\left(\lambda_{N, j, 4}-(\pi+2 \pi n)^{2}\right)\left(\Psi_{N, j, 4}, \cos (2 n+1) \pi x\right)=\left(q(x) \Psi_{N, j, 4}, \cos (2 n+1) \pi x\right),
$$




$$
\begin{aligned}
& \left(\lambda_{N, j, 4}-((2 n+1) \pi)^{2}\right)\left(\Psi_{N, j, 4}, \varphi_{n, 4}^{*}\right)-(2 n+1) \gamma_{4}\left(\Psi_{N, j, 4}, \cos (2 n+1) \pi x\right) \\
& \quad=\left(q \Psi_{N, j, 4}, \varphi_{n, 4}^{*}\right),
\end{aligned}
$$

respectively, where

$$
\gamma_{4}=\frac{8 \pi(1-\alpha)}{1+\alpha} .
$$

Instead of (16)-(19) using (77)-(80) and arguing as in the proofs of Theorem 1 and Theorem 2 we obtain the following results for $T_{4}$.

Theorem 5 If $(9)$ holds, then the large eigenvalues $\lambda_{n, j, 4}$ are simple and satisfy the following asymptotic formulas:

$$
\lambda_{n, j, 4}=((2 n+1) \pi)^{2}+(-1)^{j} \frac{\sqrt{2 \gamma_{4}}}{2} \sqrt{(2 n+1) s_{2 n+1}}(1+o(1))
$$

for $j=1,2$. The eigenfunctions $\Psi_{n, j, 4}$ corresponding to $\lambda_{n, j, 4}$ obey

$$
\Psi_{n, j, 4}=\sqrt{2} \sin (2 n+1) \pi x+O\left(n^{-1 / 2}\right) .
$$

Moreover, if there exists a sequence $\left\{n_{k}\right\}$ such that (9) holds when $n$ is replaced by $n_{k}$, then the root functions of $T_{4}$ do not form a Riesz basis.

Now suppose that

$$
\int_{0}^{1} x q(x) d x \neq 0
$$

If

$$
\frac{1}{2} s_{2 n}+B=o\left(\frac{1}{n}\right)
$$

where $B$ is defined by (34), then one can readily see from (59), (34), (38), and (50)-(53) that there exists a positive constant $K$ such that

$$
|\Delta(\lambda)|>K
$$

for $\lambda \in U(n)$ and for the large values of $n$. Therefore arguing as in the proof of Theorem 2, we obtain the following.

Theorem 6 Suppose that (81) holds. If (82) holds, then the large eigenvalues of the operator $T_{1}$ are simple. Moreover, if there exists a sequence $\left\{n_{k}\right\}$ such that (82) holds when $n$ is replaced by $n_{k}$, then the root functions of $T_{1}$ do not form a Riesz basis. Similar results continue to hold for the operators $T_{2}, T_{3}$, and $T_{4}$.

Remark 1 Since the eigenvalues $\lambda_{n, 1}$ and $\lambda_{n, 2}$ are the fixed points of (60) and (61) respectively, using the fixed point iteration one can determine these eigenvalues with arbitrary 
precision. Moreover, using these better approximations of the eigenvalues, one can also determine the better approximations for the eigenfunctions of the operator $T_{1}$. Similar results can be obtained for the operators $T_{2}, T_{3}$, and $T_{4}$.

\section{Competing interests}

The authors declare that they have no competing interests.

\section{Authors' contributions}

All authors contributed equally to the writing of this paper. All authors read and approved the final manuscript.

Received: 2 January 2014 Accepted: 28 February 2014 Published: 15 Mar 2014

\section{References}

1. Dunford, N, Schwartz, JT: Linear Operators, Part 3: Spectral Operators. Wiley-Interscience, New York (1988)

2. Kesselman, GM: On unconditional convergence of the eigenfunction expansions of some differential operators. Izv. Vuzov. Mat. 2, 82-93 (1964) (in Russian)

3. Mikhailov, VP: On Riesz bases in $\mathcal{L}_{2}[0,1]$. Dokl. Akad. Nauk SSSR 144(5), 981-984 (1962)

4. Shkalikov, AA: On the Riesz basis property of the root vectors of ordinary differential operators. Russ. Math. Surv. 34(5), 249-250 (1979)

5. Shkalikov, AA: On the basis property of the eigenfunctions of ordinary differential operators with integral boundary conditions. Vestn. Mosk. Univ., Ser. Mat. Mekh. 37(6), 12-21 (1982)

6. Kerimov, NB, Mamedov, KR: On the Riesz basis property of the root functions in certain regular boundary value problems. Math. Notes 64(4), 483-487 (1998)

7. Dernek, N, Veliev, OA: On the Riesz basisness of the root functions of the nonself-adjoint Sturm-Liouville operators. Isr. J. Math. 145, 113-123 (2005)

8. Makin, AS: Convergence of expansion in the root functions of periodic boundary value problems. Dokl. Math. 73(1), 71-76 (2006)

9. Shkalikov, AA, Veliev, OA: On the Riesz basis property of the eigen- and associated functions of periodic and antiperiodic Sturm-Liouville problems. Math. Notes 85(5), 647-660 (2009)

10. Djakov, P, Mitjagin, BS: Instability zones of periodic 1-dimensional Schrödinger and Dirac operators. Russ. Math. Surv. 61(4), 663-776 (2006)

11. Djakov, P, Mitjagin, BS: Convergence of spectral decompositions of Hill operators with trigonometric polynomial potentials. Dokl. Math. 83(1), 5-7 (2011)

12. Djakov, P, Mitjagin, BS: Convergence of spectral decompositions of Hill operators with trigonometric polynomial potentials. Math. Ann. 351(3), 509-540 (2011)

13. Djakov, P, Mitjagin, BS: Criteria for existence of Riesz bases consisting of root functions of Hill and 1D Dirac operators. J. Funct. Anal. 263(8), 2300-2332 (2012)

14. Gesztesy, F, Tkachenko, V: A Schauder and Riesz basis criterion for non-self-adjoint Schrödinger operators with periodic and antiperiodic boundary conditions. J. Differ. Equ. 253, 400-437 (2012)

15. Mamedov, KR: On the basis property in $L_{p}(0,1)$ of the root functions of a class non self adjoint Sturm-Liouville operators. Eur. J. Pure Appl. Math. 3(5), 831-838 (2010)

16. Mamedov, KR, Menken, $\mathrm{H}$ : On the basisness in $L_{2}(0,1)$ of the root functions in not strongly regular boundary value problems. Eur. J. Pure Appl. Math. 1(2), 51-60 (2008)

17. Menken, $H$, Mamedov, KR: Basis property in $L_{p}(0,1)$ of the root functions corresponding to a boundary-value problem. J. Appl. Funct. Anal. 5(4), 351-356 (2010)

18. Veliev, OA: On the nonself-adjoint ordinary differential operators with periodic boundary conditions. Isr. J. Math. 176, 195-208 (2010)

19. Veliev, OA: On the basis property of the root functions of differential operators with matrix coefficients. Cent. Eur. J. Math. 9(3), 657-672 (2011)

20. Kerimov, NB, Kaya, U: Spectral properties of some regular boundary value problems for fourth order differential operators. Cent. Eur. J. Math. 11(1), 94-111 (2013)

21. Kerimov, NB, Kaya, U: Spectral asymptotics and basis properties of fourth order differential operators with regular boundary conditions. Math. Methods Appl. Sci. (2013). doi:10.1002/mma.2827

22. Makin, AS: On spectral decompositions corresponding to non-self-adjoint Sturm-Liouville operators. Dokl. Math. 73(1), 15-18 (2006)

23. Makin, AS: On the basis property of systems of root functions of regular boundary value problems for the Sturm-Liouville operator. Differ. Equ. 42(12), 1717-1728 (2006)

24. Naimark, MA: Linear Differential Operators. Harap, London (1967)

25. Veliev, OA, Toppamuk Duman, M: The spectral expansion for a nonself-adjoint Hill operators with a locally integrable potential. J. Math. Anal. Appl. 265, 76-90 (2002)

26. Veliev, OA: Asymptotic analysis of non-self-adjoint Hill operators. Cent. Eur. J. Math. (2013) doi:10.2478/s11533-013-0305-x

10.1186/1687-2770-2014-57

Cite this article as: Nur and Veliev: On the basis property of the root functions of some class of non-self-adjoint Sturm-Liouville operators. Boundary Value Problems 2014, 2014:57 